\title{
As dinâmicas intraorganizacionais em um serviço de Saúde e Educação: análise de redes sociais
}

\section{Cintia Bincoleto Fazion}

Doutora em Administração de Empresas pela Pontifícia Universidade Católica de São Paulo. Mestre em Administração de Empresas pela Pontifícia Universidade Católica de São Paulo.

https://orcid.org/0000-0003-2565-8434

cintia_fazion@hotmail.com

\section{Luciano Antonio Prates Junqueira}

Mestre em Saúde Pública pela Universidade de São Paulo. Doutor em Administração da Saúde pela Universidade de São Paulo. Professor Titular da Pontifícia Universidade Católica de São Paulo https://orcid.org/0000-0003-1863-2463

junq@pucsp.br

Editor Científico: José Edson Lara

Organização Comitê Científico

Double Blind Review pelo SEER/OJS

Recebido em 01.10.2018

Aprovado em 22.09.2019 


\section{Resumo}

Objetivo: Analisar as dinâmicas intraorganizacionais de cooperação e trocas de conhecimento, entre profissionais que integram uma equipe multidisciplinar de um serviço de saúde e educação, de uma organização privada sem fins lucrativos. Investigou-se se há um processo de integração em rede e trocas de conhecimento e colaboração entre eles.

Método: A pesquisa, de natureza quantitativa e qualitativa, foi realizada mediante aplicação de um questionário para identificar a existência de uma rede na instituição. Caracterizou-se o perfil dos profissionais, suas competências, seu posicionamento e o tipo de conhecimento que compartilham. Os dados foram inseridos no excel e tratados no software para análise de redes. Resultados: A análise de rede identificou quatro grupos associados aos setores em que os agentes atuam e apresentou uma estrutura relativamente centralizada e com especialistas com poucas chances de compartilharem trocas de conhecimentos. A instituição apresentou uma baixa densidade de vínculos entre os profissionais e uma restrita reciprocidade nas relações entre os agentes. Isso resulta na pouca reciprocidade de relações entre os agentes.

Recomendações: Com esse cenário, recomenda-se estimular a conectividade das pessoas, por meio de ações que influenciem a interação dos profissionais em rede. Para estimular os indivíduos a atuarem em rede, entende-se importante fomentar as ações que estimulem as trocas interdisciplinares entre os profissionais, resultando em benefícios para a organização e para o público atendido.

Palavras claves: Análise de redes sociais; Agentes; Serviços de saúde e Educação; Relação intraorganizacional.

\section{The intraorganizational dynamics in a Health and Education service: Analysis of Social Networks}

\section{Abstract}

Objective: To analyze the intraorganizational dynamics of cooperation and knowledge exchange among professionals who are part of a multidisciplinary team of a health and education service of a private non-profit organization. We investigated whether there is a process of network integration and exchange of knowledge and collaboration between them.

Method: The research, quantitative and qualitative, was conducted by applying a questionnaire to identify the existence of a network in the institution. The profile of professionals, their skills, their positioning and the type of knowledge they share was characterized. Data were entered into excel and processed in the network analysis software.

Results: The network analysis identified four groups associated with the sectors in which agents operate and presented a relatively centralized structure with experts with little chance of sharing knowledge exchanges. The institution presented a low density of bonds between professionals and a restricted reciprocity in relations between agents. This results in little reciprocity of relations between agents.

Recommendations: With this scenario, it is recommended to stimulate people's connectivity through actions that influence the interaction of network professionals. In order to encourage individuals to work in a network, it is important to foster actions that encourage interdisciplinary exchanges between professionals, resulting in benefits for the organization and the public served.

Key words: Analysis of social networks; Agents; Health and education services; Interorganizational relationship. 


\section{La dinámica intraorganizacional en un servicio de salud y educación: análisis de redes sociales \\ Resumen}

Objetivo: Analizar las dinámicas intraorganizacionales de cooperación e intercambio de conocimientos entre los profesionales que forman parte de un equipo multidisciplinario de un servicio de salud y educación de una organización privada sin fines de lucro. Investigamos si hay un proceso de integración de red e intercambio de conocimiento y colaboración entre ellos. Método: La investigación, cuantitativa y cualitativa, se realizó mediante la aplicación de un cuestionario para identificar la existencia de una red en la institución. Se caracterizó el perfil de los profesionales, sus habilidades, su posicionamiento y el tipo de conocimiento que comparten. Los datos se ingresaron en Excel y se procesaron en el software de análisis de red.

Resultados: El análisis de la red identificó cuatro grupos asociados con los sectores en los que operan los agentes y presentó una estructura relativamente centralizada con expertos con pocas posibilidades de compartir intercambios de conocimiento. La institución presentaba una baja densidad de vínculos entre profesionales y una reciprocidad restringida en las relaciones entre agentes. Esto resulta en poca reciprocidad de las relaciones entre los agentes.

Recomendaciones: Con este escenario, se recomienda estimular la conectividad de las personas a través de acciones que influyen en la interacción de los profesionales de la red. Para alentar a las personas a actuar en una red, es importante fomentar acciones que fomenten los intercambios interdisciplinarios entre profesionales, lo que resulta en beneficios para la organización y el público atendido.

Palabras clave: análisis de redes sociales; Agentes; Servicios de salud y educación; Relación intraorganizacional.

\section{Introdução e contribuição da literatura}

A interação e o ambiente são fatores que moldam o desenvolvimento do indivíduo no decorrer das etapas que correspondem a sua vida: infância, adolescência e idade adulta. Esse desenvolvimento pode ser afetado por condições de vulnerabilidade econômica, que põem em risco uma vida saudável.

A população atual do Brasil é estimada em 208,6 milhões de pessoas (IBGE, 2018), das quais 6,2\% são possuidoras de algum tipo de deficiência, seja física, intelectual, auditiva ou visual, dados da Pesquisa Nacional de Saúde. Estima-se que a parcela da população com deficiência auditiva no país é de 1,1\%, sendo esse percentual maior entre pessoas sem instrução ou com pouca instrução (1,8\%) e grupo com idade acima de 60 anos (5,2\%) (PNS, 2013).

Dessa população, $0,2 \%$ a tem de nascimento e $0,9 \%$ adquiriu por doença ou acidente. Ressalta-se que, em 2013, 20,6\% da população apresentou forte grau de limitação ao ponto de não conseguir realizar atividades habituais. Em São Paulo, do total de 11.253.503 pessoas, 2.759.004 possuem pelo menos u2.ma das deficiências, mental/intelectual; auditiva; visual e motora. A população com deficiência intelectual no país está estimada em $0,8 \%$, sendo a menos 
frequente dentre as quatro deficiências mencionadas. Desse total $0,5 \%$ já possui a deficiência desde o nascimento e 0,3\% foi adquirida por acidente ou doença (PNS, 2013).

Para realizar intervenções integradas focadas no aprendizado, desenvolvimento e cuidado à pessoa com deficiência deve-se proporcionar ambientes seguros, com profissionais de excelência. Esta abordagem envolve a gestão do conhecimento no processo de promoção da qualidade do serviço prestado e de trocas de conhecimentos entre os profissionais da instituição.

A atuação em rede tem um legado histórico na compreensão da importância das atividades, "especialmente em colaboração", intraorganizacional, sendo cada vez mais a chave para sustentar vantagens dos fluxos das atividades que fortalecem a participação no processo de globalização das instituições (Hayashi, 2012, p. 286).

A necessidade da cooperação no interior das organizações conduz para um dos principais desafios, para as organizações que têm como objetivo manter-se na busca pela sustentabilidade. A troca de conhecimentos entre as pessoas tem favorecido a incorporação de novas práticas e saberes por parte dos membros de um determinado grupo, que passam a usufruir dos benefícios de se atuar em conjunto, em prol do bem comum e satisfação das necessidades coletivas.

Nesse sentido, as redes nascem num espaço informal de relações sociais, mas seus efeitos são visíveis para além desse espaço através das relações com o Estado, a sociedade e de outras instituições (Acioli, 2007). A estrutura das redes é base da formação de comunidades, além de promover o crescimento do papel das organizações não governamentais como atores influentes na sociedade civil (Junqueira, 2006).

O indivíduo não é somente o eu individual, mas também, o conjunto da sua rede relacional (Maturana, 2001) em que as relações do meio ao qual esse indivíduo pertence faz parte do processo de conhecimento no qual não se dissocia a ação da prática. O conhecimento, por sua vez, é o processo de formação dos profissionais que se faz pela convivência social das organizações, ensinando e aprendendo, não somente no ambiente de trabalho, mas, sobretudo, nas redes internas e externas, trabalhar em rede é um processo que edifica colaborações entre as pessoas.

A contemporaneidade exige da organização a compreensão do contexto sob o qual está ancorada, os sistemas e subsistemas dos quais faz parte. As redes que se consolidam no seu interior e aquelas que conduzem a um quadro integral, tomando por base a intensidade das relações que ela desenvolve com o meio (Fialho; Moreno, 2015). 
Essas mudanças nos cenários têm sido mais acentuadas, a partir dos anos 2000, quando surge novas formas de produção e organização. A união desses esforços, em torno de um objetivo comum, converge para uma maior atenção de organismos de cooperação técnica, assim como de órgãos de financiamento e incentivo ao crescimento social e econômico, que passam a definir políticas diversificadas para a construção de redes de cooperação ou novas formas que possam favorecer o incremento da produção em nível local (Teixeira; Beber; Grzybovski, 2008).

Nessa perspectiva é possível salientar que as redes estão presentes em todas as esferas da vida social, ou seja, onde existe a construção de relações de organizações em redes. Tais redes tomam forma por meio de indivíduos, de organizações, de troca de informações, comunicação e até mesmo de pactos sociais. Esta lógica das relações incorpora o primado da cooperação que tem sua lógica pautada na interação (Fialho; Moreno, 2015).

Assim, as redes de cooperação estabelecidas entre organizações sociais privilegiam o nível complexo dos conhecimentos produzidos e dinamizam a sua apropriação para a oferta de bens e serviços que satisfaçam as necessidades da sociedade (Teixeira; Beber; Grzybovski, 2008).

As redes estabelecem acordos de cooperação, de alianças e de reciprocidade entre as pessoas e as organizações. "Essas novas práticas de cooperação constituem um meio de encontrar saídas para intervir na realidade social complexa” (Junqueira, 2000, p. 40).

Esse processo de interação e atuação em grupo perfaz a ideia da união em rede. A percepção conjunta de resolver problemas comuns que afetam ou dizem respeito a uma determinada categoria de indivíduos ou organizações, de onde se vislumbra por meio da cooperação a possibilidade de resolvê-los, ou mesmo, de desenvolver novas habilidades, novos produtos, processos mais eficazes e ideias melhores. Condições que demonstram a existência de um processo de articulação e integração entre os diferentes atores envolvidos.

As redes sociais são redes de interação contínua entre atores - indivíduos ou organizações, diante das quais ocorre o intercâmbio de diferentes conteúdos abstratos como informação, sentimentos, conselhos, ou coisas concretas como bens e serviços. Tal interação pode suceder tanto em espaços físicos como em elos virtuais, embora em ambos os contextos seja indispensável o envolvimento das partes para concretização de um objetivo em comum, ou então, os objetivos não seriam atingidos (Santanita, 2015), cuja característica essencial é a interação entre os membros pertencentes à mesma rede, podendo evoluir com o tempo, ou se dissipar (Snijders, 2010). 
No âmbito desta discussão, a ideia de rede permite o compartilhamento de práticas e recursos, propiciando uma ação solidária e o fortalecimento dos atores conectados, que permite circular elementos materiais ou imateriais entre eles. Analisar as relações dos profissionais que integram a equipe de prestação de serviços interdisciplinares do serviço de saúde e educação, por meio da análise de rede é possível aumentar a cooperação entre os profissionais e identificar oportunidades de treinamento e trocas de saberes entre os mesmos.

Portanto, este estudo tem por objetivo diagnosticar, por meio da análise de rede, a cooperação entre os profissionais que integram a equipe interdisciplinar no serviço de saúde e educação.

\section{Método}

A pesquisa é de natureza quantitativa e qualitativa e tem como objeto as relações sociais entre os atores de uma Instituição, caracterizada como pessoa jurídica de direito privado, sem fins lucrativos e localizada na cidade de São Paulo.

Essa unidade de saúde e educação é vinculada a uma Universidade de pesquisa e extensão e, administrativamente, mantida por uma Fundação, sem fins lucrativos. Atua com foco no atendimento clínico a pessoas com alterações de audição, voz e linguagem, na educação de surdos e a com deficiência intelectual. Igualmente, atua na assessoria à acessibilidade dos deficientes auditivos, na empregabilidade de surdos e no desenvolvimento de pesquisas. Por ser uma instituição sem fins lucrativos, além da população convencional, prioriza famílias economicamente desfavorecidas e beneficia pessoas de todas as faixas etárias.

Essas atividades geram impacto social nos campos da educação, empregabilidade e acessibilidade de surdos, da saúde auditiva, da voz e da linguagem de crianças, jovens, adultos e idosos; na formação e especialização de alunos e profissionais de diversas áreas e na criação e disseminação do conhecimento científico, mobilização que gera benefício e novos níveis de desenvolvimento para a sociedade.

O diferencial da Instituição é a concentração e consolidação de três eixos de atuação: atendimento clínico, formação educacional e pesquisa. Dispõe de profissionais nas áreas de medicina, fonoaudiologia, psicologia, pedagogia e linguística que compõem a equipe proporcionando atendimento multidisciplinar.

A pesquisa foi realizada mediante a aplicação de um questionário para verificar a conformação de uma rede na instituição. O questionário abrange dados demográficas (idade, 
escolaridade, profissão, cargo na instituição, etc.) e as relações que os atores estabelecem entre si no seu cotidiano profissional, como: quem você conhece, com quem você já atuou, para realizar qual atividade.

A participação dos respondentes permitiu mapear as relações entre os profissionais cadastrados na instituição. No início da pesquisa foi informado o tema e objetivo do estudo, solicitando sua concordância em responder ao questionário proposto.

Os dados coletados foram tratados como informações confidenciais, sem a identificação dos respondentes, sendo garantido o anonimato. A identificação das respostas foi feita por código ${ }^{1}$ (letra, número ou nome fictício), ou seja, sua identidade foi protegida.

Esta pesquisa analisa as relações entre os diferentes atores sociais, cujo grau de conectividade pode se dar em menor ou maior intensidade e frequência através da análise de redes sociais, compreendida como uma maneira de investigar como estão construídas as estruturas sociais a partir de dados relacionais dos atores envolvidos, com precendentes nas teorias dos grafos e na sociometria (Wasserman \& Faust, 1994; Bufrem \& Prates, 2005; Cordeiro, 2009).

A realização da pesquisa ocorreu em duas etapas. A primeira se deu pela aplicação do questionário eletrônico estruturado para mapeamento de redes por meio da ferramenta SurveyMonkey ${ }^{2}$. O instrumento de pesquisa foi enviado aos respondentes através do e-mail da Instituição. Tendo em vista a baixa adesão dos respondentes, via internet, decidiu-se aplicar o questionário via malote, numa segunda etapa, perfazendo um total de 92 respondentes. $\mathrm{O}$ período da coleta foi de junho a outubro de 2016.

Nesta pesquisa foi caracterizado o perfil dos profissionais, identificando a sua área de formação, experiência profissional e competências específicas, além das relações entre esses profissionais que atuam no serviço de saúde e educação.

Os dados obtidos passaram por um tratamento prévio antes da etapa de análise, sendo catalogados, sistematizados e tabulados no Excel. Este procedimento de tratamento dos dados visou eliminar duplicidade e gerar uma base de dados única para importação para o software de análise.

Ao final os dados foram inseridos para análise no software, ORA.NetScenes (Carley, 2011). O software utiliza, na sua base, elementos da psicologia social, da pesquisa operacional

\footnotetext{
${ }^{1}$ A letra utilizada é $\mathrm{A}=$ Agente. A terminologia agente é atribuída aos profissionais da Instituição analisada.

2 Disponível em: https://pt.surveymonkey.com/
} 
e da teoria da administração, para identificar os laços que unem pessoas e as tarefas realizadas em rede (Carley; Reminga, 2004; Pinto, 2008).

\section{Análises dos resultados}

\section{Perfil dos Respondentes}

Os colaboradores da Instituição foram analisados com base nos critérios definidos na metodologia de pesquisa. Foram identificados aqueles com maior número de indicações pelos respondentes da pesquisa, o que permitiu traçar o mapa da relação profissional existente entre eles. Além disso, tornou-se possível, a apresentação do perfil dos colaboradores, nas atividades de atuação, o nível de conhecimento e o interesse pelas atividades da área de trabalho.

A pesquisa foi aplicada considerando o universo de 131 colaboradores que compõem o quadro total de funcionários. Destes, 92 (70,2\%) responderam à pesquisa, no entanto, tivemos alguns funcionários que não responderam a algumas perguntas, por esse motivo, os números por item será alterado de acordo com o número de respondentes.

Dentre o total de respostas, 90 (noventa) indivíduos responderam qual as áreas de atuação estão vinculadas. A maioria, 44,9\% (40 indivíduos), pertence ao Instituto Educacional São Paulo, 24,7\% (22 indivíduos) pertencem ao setor Administrativo, 23,6\% (21 indivíduos) pertencem ao setor Clínica de Audição, Voz e Linguagem; e 20,2\% pertencem ao setor Centro de Audição na Criança.

O perfil desses respondentes contempla as seguintes variáveis: escolaridade, tempo de casa, tempo de formado. Quanto à escolaridade dos respondentes (tabela 1) o dominante na Instituição são profissionais de curso superior completo $(45,7 \%)$ seguido da pós-graduação $(19,6 \%)$, totalizando $65,3 \%$.

\section{Tabela 1}

Número de Respondentes por Escolaridade

\begin{tabular}{rcr}
\multicolumn{1}{|c}{ Escolaridade } & Respondentes & \multicolumn{1}{c}{$\%$} \\
\hline Fundamental & 1 & $1,1 \%$ \\
Médio & 6 & $6,5 \%$ \\
Superior & 42 & $45,7 \%$ \\
Superior Incompleto & 4 & $4,3 \%$ \\
Pós-Graduação - Lato Sensu & 18 & $19,6 \%$ \\
Mestrado - Stricto Sensu & 13 & $14,1 \%$ \\
Doutorado - Stricto Sensu & 5 & $5,4 \%$ \\
ND & 3 & $3,3 \%$ \\
Total Geral & $\mathbf{9 2}$ & $\mathbf{1 0 0 , 0 \%}$ \\
\hline & &
\end{tabular}

2), a maioria dos

\section{Total Geral} $3,3 \%$

tempo de casa (tabela respondentes possui

de 01 a 10 anos de contrato junto à instituição, correspondendo a 30,4\% do total. 


\section{Tabela 2}

Número de Respondentes por Tempo de Casa

\begin{tabular}{rcc} 
Tempo de Casa & Respondentes & \multicolumn{1}{c}{$\%$} \\
Menos de 1 ano & 12 & $13,0 \%$ \\
01 a 10 anos & 28 & $30,4 \%$ \\
1120 anos & 13 & $14,1 \%$ \\
21 a 30 anos & 16 & $17,4 \%$ \\
Acima de 30 anos & 20 & $21,7 \%$ \\
ND & 3 & $3,3 \%$ \\
\hline Total Geral & $\mathbf{9 2}$ & $\mathbf{1 0 0 , 0 \%}$
\end{tabular}

No que diz respeito ao tempo de formação (tabela 3), a maioria dos respondentes está concentrada na faixa de 11 a 20 anos de tempo de formado, que representa $25 \%$ dos colaboradores. A esses agregam os seus os com até 10 anos de formado e a representatividade totaliza $48,9 \%$.

Tabela 3
Número de Respondentes por Tempo de Formado
\begin{tabular}{rcc} 
Tempo de Formado & Respondentes & $\%$ \\
\hline Até 10 anos & 22 & $23,9 \%$ \\
11 a 20 anos & 23 & $25,0 \%$ \\
21 a 30 anos & 13 & $14,1 \%$ \\
Acima de 30 anos & 21 & $22,8 \%$ \\
ND & 13 & $14,1 \%$ \\
\hline Total Geral & $\mathbf{9 2}$ & $\mathbf{1 0 0 , 0 \%}$
\end{tabular}

Com relação à área de graduação (tabela 4), do total de 92 respondentes, 81 identificou sua área de formação. A maioria dos respondentes possui graduação em Fonoaudiologia e Pedagogia que representam $53,1 \%$ e se agregada a categoria educação $(9,9 \%)$ este total passa para $63,1 \%$. 


\section{Tabela 4}

Número de Respondentes por Área de Formação

\begin{tabular}{rcc} 
Área de Formação & Respondentes & \multicolumn{1}{c}{$\%$} \\
\hline Administração & 5 & $6,2 \%$ \\
Biblioteconomia & 1 & $1,2 \%$ \\
Ciência Contábeis & 2 & $2,5 \%$ \\
Design, Publicidade, Marketing & 1 & $1,2 \%$ \\
Direito & 1 & $1,2 \%$ \\
Distúrbio da Comunicação & 2 & $2,5 \%$ \\
Economia & 2 & $2,5 \%$ \\
Educação & 8 & $9,9 \%$ \\
Educação Física & 5 & $6,2 \%$ \\
Engenharia & 1 & $1,2 \%$ \\
Fonoaudiologia & 25 & $30,9 \%$ \\
Fundamental & 1 & $1,2 \%$ \\
Geografia & 1 & $1,2 \%$ \\
História & 1 & $1,2 \%$ \\
Informática & 1 & $1,2 \%$ \\
Letras & 2 & $2,5 \%$ \\
Medicina & 1 & $1,2 \%$ \\
Pedagogia & 18 & $22,2 \%$ \\
Psicologia & 2 & $2,5 \%$ \\
Serviço Social & 1 & $1,2 \%$ \\
Total Geral & $\mathbf{8 1}$ & $\mathbf{1 0 0 , 0 \%}$
\end{tabular}

Dos respondentes, $11(12,4 \%)$ atuam em mais de um setor (tabela 5), o que representa a existência de 24 relações em rede. Ao atuar em diversos setores, um agente pode atuar como ponte relacional entre os setores, desde que tenha tempo para nutrir relações em ambos os setores.

\section{Tabela 5}

Número de Respondentes por Área de Atuação

\begin{tabular}{|c|c|c|c|c|c|c|}
\hline ÁREA & AGENTE & Administrativo & Centro & Clínica de & Instituto & Total \\
\hline Assistência Social & A058 & & 1 & 1 & 1 & 3 \\
\hline Fonoaudiologia & A075 & 1 & 1 & 1 & & 3 \\
\hline Fonoaudiologia & A052 & & 1 & 1 & & 2 \\
\hline Fonoaudiologia & A055 & & 1 & 1 & & 2 \\
\hline Fonoaudiologia & A053 & & 1 & 1 & & 2 \\
\hline Medicina & A057 & & 1 & 1 & & 2 \\
\hline Administrativa & A019 & 1 & & 1 & & 2 \\
\hline Fonoaudiologia & A012 & & 1 & 1 & & 2 \\
\hline Coordenação & A064 & 1 & 1 & & & 2 \\
\hline Fonoaudiologia & A051 & & 1 & 1 & & 2 \\
\hline Administrativa & A074 & 1 & & & 1 & 2 \\
\hline \multicolumn{2}{|c|}{ Total Geral } & 4 & 9 & 9 & 2 & 24 \\
\hline
\end{tabular}

Os agentes indicados (Tabela 5) estão em 4 setores: Centro de Audição na Criança CeAC (Setor 02 - S02) e na Clínica de Audição, Voz e Linguagem (Setor 03 - S03). Esses setores são os que mais concentram profissionais em comum com outros serviços ( 9 agentes cada). Os setores Administrativo (Setor 01 - S01) e Instituto Educacional São Paulo - IESP 
(Setor 04 - S04) apresentaram menor concentração de profissionais. Destacam-se os agentes A058 e A075, ambos atuam em três diferentes setores, como releva a Figura 1. Esse gráfico permite visualizar como ocorre a atuação desses profissionais em rede.

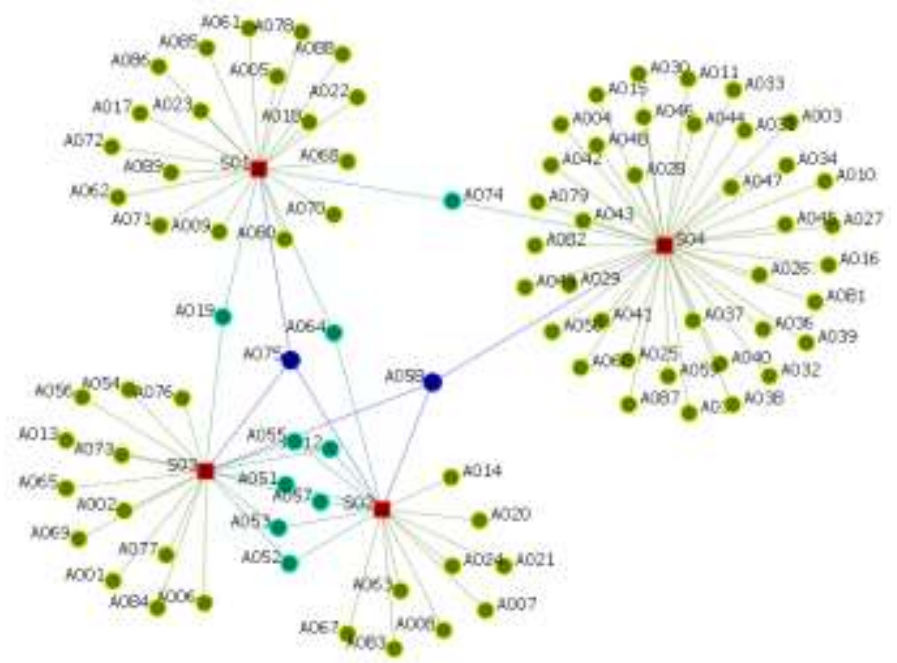

Figura 1: Respondentes por Área de Atuação

A cor dos nós entre os agentes é em função do grau de proximidade, por área de atuação, que indica a intensidade e onde existe maior chance de haver vínculo entre os profissionais.

\section{Relações entre os Profissionais Agentes Indicados na Sua Área de Atuação}

Neste item a pergunta solicitou que os respondentes indicassem duas pessoas, que na Instituição, procura quando tem dúvidas. A recomendação foi que o respondente informasse os dois primeiros nomes que viessem à sua mente.

A Figura 2 ilustra a multiplicidade e a complexidade das pessoas indicadas como muito procuradas pelas áreas de atuação quando o respondente tem dúvidas. Os agentes A089 (profissional da área administrativa) e A057 (profissional da área médica), foram indicados por 64 respondentes das diferentes áreas de atuação da Instituição, ambos possuem muita ou algum tipo de ligação com os demais. 


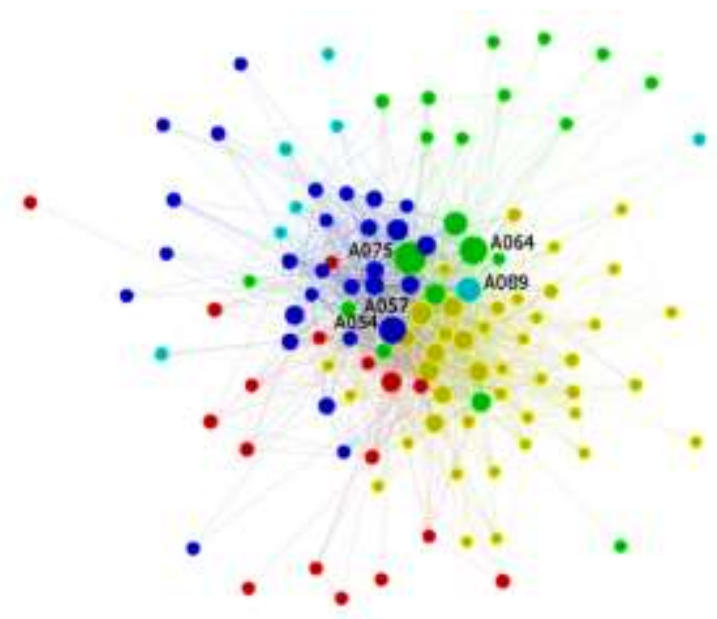

Figura 2 - Agentes Indicados por Tema

As métricas (Tabela 7) apontam uma rede com 127 agentes indicados, e 1.283 relações entre estes agentes, com uma baixa densidade de vínculos (8\%). A distância relacional média entre os agentes é baixa (2,7 graus de separação), há pouca reciprocidade de relações $(8,2 \%)$. É uma rede relativamente centralizada (grau de hierarquia de 73,9\%), o que indica a concentração em alguns poucos especialistas. A rede seria muito mais rica e resiliente se as reciprocidades fossem menos concentradas, com maior reciprocidade de procuras e trocas de conhecimentos entre os agentes.

\begin{tabular}{lr}
$\begin{array}{lr}\text { Tabela } 7 \\
\text { Métricas dos Agentes Indicados por }\end{array}$ & Tema \\
\multicolumn{1}{|c}{ Medida } & \multicolumn{1}{c|}{ Valor } \\
\hline Número de linhas & 127.000 \\
Contagem de colunas & 127.000 \\
Contagem de links & 1.283 .000 \\
Densidade & 0.080 \\
Reciprocidade & 0.082 \\
Comprimento do caminho característico & 2.722 \\
Coefiente de agrupamento & 0.243 \\
Hierarquia Krackhardt & 0.739
\end{tabular}

Abaixo (tabela 8) são identificados os temas pelos quais as cinco pessoas foram mais procuradas pelos respondentes da Instituição, quando têm dúvidas sobre as atividades a serem desempenhadas. As pessoas indicadas representam 21,8\% das 1.283 indicações atribuídas por todos os respondentes. 


\section{Tabela 8}

Agentes Indicados por Tema

\begin{tabular}{lrr}
\hline AGENTE / TEMA & QUANT & \multicolumn{1}{c}{$\%$} \\
\hline A089 & $\mathbf{6 4}$ & $\mathbf{7 1 , 1 \%}$ \\
\hline Administração & 60 & $66,7 \%$ \\
Triagem auditiva neonatal & 2 & $2,2 \%$ \\
Desenvolvimento institucional & 2 & $2,2 \%$ \\
A057 & $\mathbf{6 4}$ & $\mathbf{7 1 , 1 \%}$ \\
\hline Consulta médica & 59 & $65,6 \%$ \\
Atendimento clínico & 3 & $3,3 \%$ \\
Triagem auditiva neonatal & 2 & $2,2 \%$ \\
A075 & $\mathbf{5 9}$ & $\mathbf{6 5 , 6 \%}$ \\
\hline Atendimento clínico & 27 & $30,0 \%$ \\
Seleção e adaptação de aparelhos auditivos & 21 & $23,3 \%$ \\
Projetos de pesquisa & 11 & $12,2 \%$ \\
A054 & $\mathbf{4 9}$ & $\mathbf{5 4 , 4 \%}$ \\
\hline Atendimento bilingue & 25 & $27,8 \%$ \\
Terapia fonoaudiológica & 12 & $13,3 \%$ \\
Evolução do uso da língua de sinais pelos surdos & 12 & $13,3 \%$ \\
A064 & $\mathbf{4 4}$ & $\mathbf{4 8 , 9 \%}$ \\
\hline Administração & 17 & $18,9 \%$ \\
Projetos de pesquisa & 16 & $17,8 \%$ \\
Atendimento clínico & 11 & $12,2 \%$
\end{tabular}

O profissional A089 foi indicado nos temas administração, triagem auditiva neonatal e desenvolvimento institucional. O profissional A057 foi procurado pelos temas consulta médica, atendimento clínico e triagem auditiva neonatal. Ambos possuem muita ou algum tipo de ligação com os demais. Na sequência o profissional A075 foi indicado principalmente nos temas atendimento clínico, seleção e adaptação de aparelhos auditivos e projetos de pesquisa. Esses profissionais representam 66,7\% dos indicados, acredita-se que esses profissionais possam ser líderes nas suas áreas de atuação, consequentemente, considerados especialistas nos temas de conhecimento.

Essas medidas indicam que esse grupo possui um maior potencial para compartilhar conhecimentos. Conforme menciona Rovere (1999), a disseminação do conhecimento é uma das estratégias para aprimoramento de vínculos entre os atores da rede, além do que a indicação é uma manifestação de reconhecer a existência do outro, sua importância e posição na rede.

Através dessas indicações pode haver proposições e ações que figuram a conectividade entre os indivíduos. Os profissionais com pouca ou nenhuma indicação são aqueles que se supõe que não sejam multiplicadores.

\section{Agentes Indicados na Realização das Atividades Profissionais}


A questão em tela buscou indicações das pessoas com os quais os respondentes mantém maior contato (pessoal, por telefone, e-mail etc.) sobre os processos de trabalho que desempenha, exceto seu superior imediato. A maior concentração de indicados se deu aos profissionais A089 e A060 (tabela 9), o primeiro vinculado à escola e o segundo à área administrativa; estes foram os mais indicados pelos agentes como sendo as pessoas com quem têm maior contato. Cada um deles se relaciona com 19,1\% dos respondentes.

\section{Tabela 9}

Agentes Indicados nas Atividades Profissionais

\begin{tabular}{lrc} 
AGENTE & QUANT & \% \\
\hline A089 & 17 & $19,1 \%$ \\
A060 & 17 & $19,1 \%$ \\
A075 & 14 & $15,7 \%$ \\
A078 & 13 & $14,6 \%$ \\
A043 & 11 & $12,4 \%$ \\
A040 & 9 & $10,1 \%$
\end{tabular}

A indicação dos profissionais A089 e A060 aponta que ambos desempenham um papel de interlocução entre os diferentes grupos, dinamizando o acesso a informações e contatos por outros meios (Figura 3).

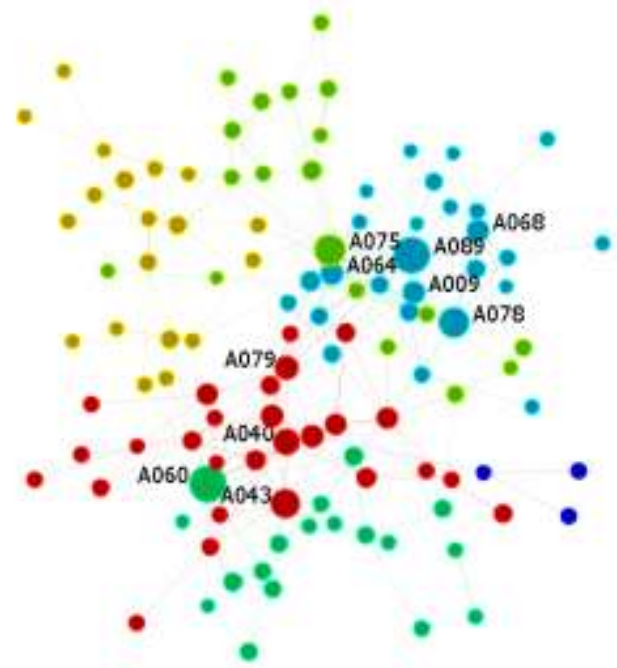

Figura 3 - Agentes Indicados nas Atividades Profissionais

Esse contato entre os profissionais mostra como o compartilhamento do conhecimento, informações e difusão fluem pela rede interna e externa a organização. Esses profissionais produzem elementos que podem possibilitar influenciar a rede positivamente ou negativamente, além de criar oportunidades para corrigir e melhorar o ambiente organizacional (Sant'ana, 2011). 


\section{Agentes Indicados nas Relações Pessoais}

Nesse item apresenta-se as relações pessoas em que cada respondente citou os colaboradores da Instituição com os quais têm maior vínculo ou que conhece com alguma profundidade (pessoas com quem já estudou, trocou experiências ou já conviveu fora dos encontros da organização). Os agentes A09 e A045 (tabela 10) foram indicados por 10,1\% dos respondentes que conhecem com alguma profundidade.

\section{Tabela 10}

Agentes Indicados nas Relações Pessoais

\begin{tabular}{lrr} 
AGENTE & QUANT & \multicolumn{1}{c}{$\%$} \\
\hline A009 & 9 & $10,1 \%$ \\
A045 & 9 & $10,1 \%$ \\
A040 & 8 & $9,0 \%$ \\
A037 & 7 & $7,9 \%$ \\
A068 & 7 & $7,9 \%$ \\
A011 & 6 & $6,7 \%$ \\
A027 & 6 & $6,7 \%$ \\
A030 & 6 & $6,7 \%$ \\
A022 & 5 & $5,6 \%$ \\
A078 & 5 & $5,6 \%$ \\
A060 & 5 & $5,6 \%$ \\
A049 & 5 & $5,6 \%$
\end{tabular}

As indicações podem ser visualizadas no Figura 4, que sinaliza os agentes A09, A045 e A040 como sendo os profissionais de maior grau de procura. São pessoas que se relacionam bem e geram vínculos que podem extrapolam o ambiente de trabalho, propiciando um maior volume de trocas informais, gerando relações pessoais fora e dentro da organização.

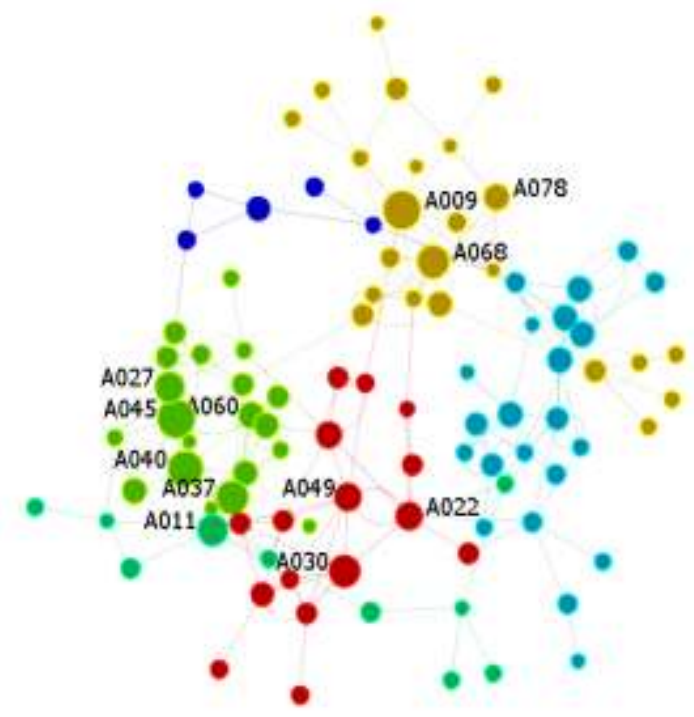

Figura 4 - Agentes Indicados nas Relações Pessoais

O agente A09 está vinculado à área administrativa (analista administrativo) da Instituição e possui 42 anos de tempo de casa. O segundo e terceiro agentes mais indicados, 
A045 e A040 respectivamente, são professores especializados em deficiência auditiva; o primeiro trabalha há 26 anos e o segundo há 19 anos. Esta rede tem uma densidade de 2,0\%, com reciprocidade nas relações de 13,9\%, um grau de formação de 'panelinhas' de 16,7\%, e eficiência na comunicação de $97,2 \%$. Isto indica que as relações deste tipo são pouco densas, concentradas em algumas pessoas e grupos e também assimétricas.

As relações tendem a ocorrer mais no âmbito profissional do que no pessoal, não se estendendo para além do ambiente de trabalho e não havendo indicação de relação que possuam vínculo com maior proximidade pessoal. Há provavelmente coleguismo profissional, mas, pouca amizade.

\section{Relações entre os Temas - Profissionais X Serviço X Atividade (Conhece e Atua)}

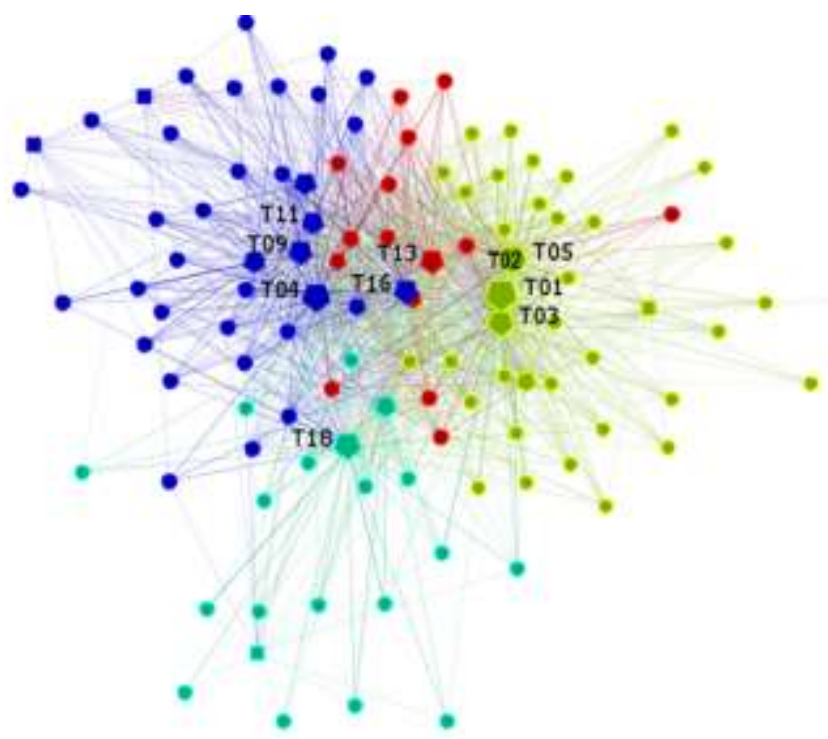

Figura 5 - Relações dos Grupos de Interesse

Identificadas 4 comunidades por setor e tema de interesse (conhece, atua, gosta), (Figura 5). O trabalho e atuação tendem a criar 'panelas' temáticas. A primeira e maior comunidade, formada por 34 agentes, é ligada principalmente ao IESP, e têm como foco o estudo, formação e atendimento de surdos e linguagem de sinais:

GRUPO 01

T05

T03

T02

T01

S04
34

Atendimento bilíngue

Evolução do uso da língua de sinais pelos surdos

Formar ouvintes em LIBRAS

Educar e formar surdos

IESP 
A segunda comunidade, com 28 agentes, é ligada ao CeAC e CAVL, focada na pesquisa, terapia, avaliação, triagem e atendimento:

$\begin{array}{ll}\text { GRUPO 02 } & \\ \text { T16 } & \text { Projetos de pesquisa } \\ \text { T11 } & \text { Terapia fonoaudiológica } \\ \text { T10 } & \text { Seleção e adaptação de aparelhos auditivos } \\ \text { T09 } & \text { Avaliação audiológica } \\ \text { T08 } & \text { Triagem auditiva neonatal } \\ \text { T07 } & \text { Consulta médica } \\ \text { T04 } & \text { Atendimento clínico } \\ \text { S03 } & \text { Clínica de Audição, Voz e Linguagem } \\ \text { S02 } & \text { Centro Audição na Criança (CeAC) }\end{array}$

O terceiro grupo, com 17 agentes, tem vínculo principal com o Setor de Administração e foco em temas correlatos (administração e desenvolvimento institucional):

GRUPO 03

T18

T17

S01
17

Administração

Desenvolvimento institucional

Administrativo

O quarto grupo, com 10 agentes, mostra preponderância nos temas que seguem:

GRUPO 04

T15

T14

T13

$\mathrm{T} 12$

T06
10

Estudos sócio econômicos dos usuários

Oficina terapêutica

Grupos de pais

Terapia psicológica

Terapia ocupacional

Apesar do grupo verde lima ter número maior de agentes, é o grupo azul que intermedia outras relações com os demais grupos e maior número de atividades. 


\section{Relações Intergrupos por Setor (Agente X Serviço X Relações: Conhecimento, Contato Profissional, Relações Pessoais)}

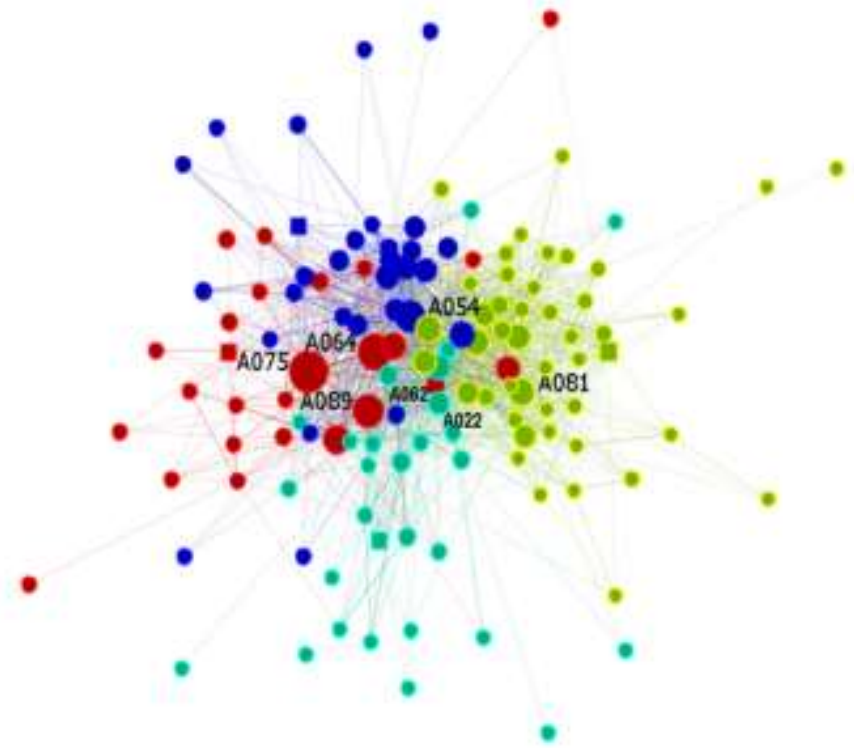

Figura 6 - Relações Intergrupos por Setor

Foram identificadas, pelo método de Newman, 4 grupos, claramente associadas aos setores em que os agentes atuam (Figura 6). Isto indica que as pessoas tendem a se relacionar apenas com outros próximos no mesmo setor, tendo como extremo a formação de 'ilhas' relacionais ou 'feudos' departamentais. Nesses grupos pode-se dispender energia em conflitos ou manutenção de posições, ao invés de trocas de informações para disseminar conhecimentos.

O primeiro grupo (Tabela 11) conta com 45 agentes. É interessante notar que os agentes mais pontuados em expertise (Figura 4) não são os mais pontuados em contato profissional (Figura 5) ou relações pessoais (Figura 6), exceto os agentes A037 e A060, que tiveram indicações elevadas nas três dimensões, expertise, contato profissional e pessoal. Isto aponta que, nesta Instituição, as relações tendem a ser bastante direcionadas, ou seja, obter informação técnica ou resolver questões cotidianas e de trabalho. 


\section{Tabela 11}

\begin{tabular}{|c|c|c|c|c|}
\hline \multirow{2}{*}{$\begin{array}{l}\text { S04 } \\
\text { AGENTE }\end{array}$} & \multicolumn{4}{|c|}{ Instituto Educacional São Paulo } \\
\hline & GRUPO & 13 & 14 & 15 \\
\hline A057 & 1.0 & 65 & 1 & 2 \\
\hline A081 & 1.0 & 62 & 5 & 4 \\
\hline A036 & 1.0 & 53 & 6 & 4 \\
\hline A101 & 1.0 & 52 & 2 & 2 \\
\hline A037 & 1.0 & 47 & 6 & 7 \\
\hline A107 & 1.0 & 43 & & 3 \\
\hline A100 & 1.0 & 39 & 2 & 4 \\
\hline A060 & 1.0 & 24 & 17 & 5 \\
\hline A043 & 1.0 & 14 & 11 & 4 \\
\hline A040 & 1.0 & 10 & 9 & 8 \\
\hline A011 & 1.0 & 8 & 3 & 6 \\
\hline A049 & 1.0 & 3 & 4 & 5 \\
\hline A045 & 1.0 & 2 & 3 & 9 \\
\hline A027 & 1.0 & 1 & 3 & 6 \\
\hline A030 & 1.0 & 1 & 3 & 6 \\
\hline
\end{tabular}

A segunda comunidade está mais associada à Clínica de Audição, Voz e Linguagem. Neste grupo, a maior pontuação em expertise (Figura 4) corresponde também à maior nota de contato (Figura 5), mas, as notas de profundidade (Figura 6) são baixas para todos, indicando um grupo que convive pouco fora do ambiente de trabalho.

Esta comunidade (Tabela 12) tem agentes (A054, A096, A117) que geram vinculação de mais de um tipo, sendo que a busca de conhecimento se dá no decorrer da própria relação cotidiana de trabalho, mas não se estende fora do expediente, o que reitera as inferências do grupo anterior

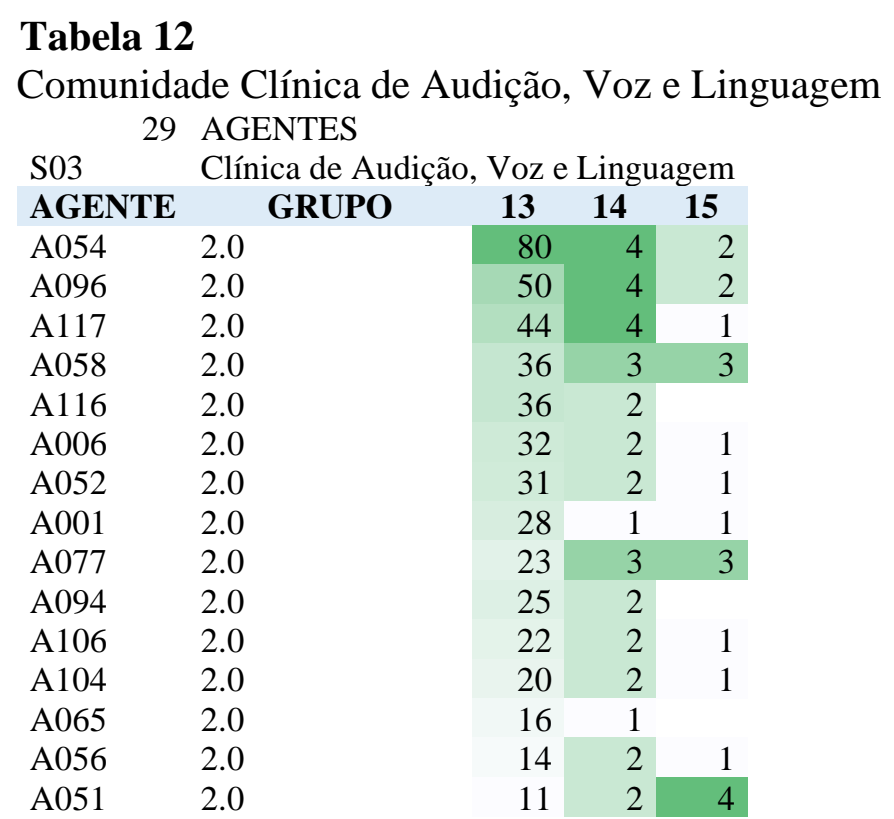


A terceira comunidade (Tabela 13) liga-se principalmente ao Administrativo. É nítida a separação entre os mais pontuados no Figura 4 (A022, A062), Figura 5 (A078) e Figura 6 (A009, A068).

\begin{tabular}{ll|r|r|r|}
\multicolumn{5}{c}{ Tabela 13 } \\
Comunidade Administrativo \\
28
\end{tabular}

A quarta comunidade (tabela 14) liga-se principalmente ao CeAC, sendo composta por 25 agentes. As pontuações entre as Figuras 4, 5 e 6 são mais próximas que nos outros grupos e concentradas nos agentes A075, A064, e A089, o que indica um grupo mais concentrado e com relacionamento mais próximo que os demais. É uma comunidade pequena, mas que provavelmente tem vínculos intensos e duradouros, parcerias e suporte mútuo.

$\begin{array}{llrrr}\text { Tabela 14 } \\ \text { Comunidade Centro Audição na Criança } \\ \text { 2502 } & \text { AGENTES } \\ \text { Centro Audição na Criança (CeAC) } \\ \text { AGENTE } & \text { GRUPO } & \mathbf{1 3} & \mathbf{1 4} & \mathbf{1 5} \\ \text { A075 } & 4.0 & 113 & 14 & 4 \\ \text { A064 } & 4.0 & 91 & 7 & 4 \\ \text { A089 } & 4.0 & 70 & 17 & 1 \\ \text { A083 } & 4.0 & 64 & 1 & 2 \\ \text { A053 } & 4.0 & 48 & 5 & 2 \\ \text { A079 } & 4.0 & 41 & 7 & \\ \text { A023 } & 4.0 & 16 & 1 & \\ \text { A097 } & 4.0 & 9 & & 4 \\ \text { A055 } & 4.0 & 5 & 5 & 3 \\ \text { A098 } & 4.0 & 9 & & 2\end{array}$

A análise entre os grupos parece indicar que não há integração em rede entre os profissionais. Talvez seja importante pensar qual o meio para tornar mais compartilhado, no âmbito da organização, a interação entre os profissionais para trocar experiências e conhecimentos. A saída seria criar espaços de diálogos com temas de interesse em comum. 


\section{Considerações Finais}

A caracterização de uma rede é marcada por apoio espontâneo, cooperação e conexão entre os indivíduos, atuando como organismo vivo, buscando compreender e interagir.

A rede analisada apresentou uma estrutura cuja identificação é a centralização em alguns especialistas que compartilham conhecimentos. $\mathrm{O}$ funcionamento da rede não é criado em torno de um profissional, mas por todos os integrantes da instituição e requer que se constituam em torno de valores, sentimentos e ideias mobilizadoras.

$\mathrm{Na}$ análise da rede constatou-se que para estimular os indivíduos a atuarem em rede seria importante fomentar as ações para conectar as pessoas da instituição; estimular as trocas interdisciplinares entre os profissionais, que resultem em benefícios para a população atendida.

Daí a necessidade de fomentar uma cultura na qual o indivíduo se perceba não apenas como parte da realidade organizacional, mas, também, como integrante de um todo, de um sistema, no qual tanto as experiências profissionais como àquelas de cunho pessoais possam ser aglutinadas em prol de algo maior. Isso deve ocasionar a base para mudar os vínculos existentes e contribuir para a maximização da integração em rede entre os grupos atuantes na instituição.

Esse processo poderia proporcionar maior volume de trocas interdisciplinares, na medida em que um ambiente de compartilhamento e troca de conhecimentos e ideias, poderia mudar a percepção de individualidade em prol de um ambiente colaborativo.

Todavia, nesse processo de transição é pertinente que se tenha, a todo momento a presença de diálogo constante e a realização de encontros temáticos, que sejam do interesse de todos e que possam reforçar os vínculos existentes.

Provavelmente, esse novo cenário a emergir no ambiente organizacional possibilitará a busca por conhecimentos subsidiando o fortalecimento dos laços existentes entre os profissionais da organização. A Instituição pesquisada apresentou uma baixa densidade de vínculos e uma distância média entre os agentes relativamente reduzida. Isso resulta na pouca reciprocidade de relações entre os agentes. Assim, recomenda-se estimular a conectividade das pessoas, por meio de ações que influenciem a interação dos profissionais em rede.

Ao discutir a rede deve-se ter presente que ela surge a partir de um reflexo da interação social existente entre indivíduos da organização, a partir de objetivos, valores, sentimentos e ideias compartilhadas coletivamente. $\mathrm{O}$ incentivo a seu desenvolvimento surge a partir de um elemento essencial, os 'tecelões ativos', que são os indivíduos que auxiliam na mobilização e integração dos participantes da instituição. 
Quanto maior e mais amplo o processo colaborativo na organização, mais redes poderão ser tecidas. Como consequência, mais duradouras serão as relações e mais sólidas as redes e subredes. Uma das ideias seria maximizar a integração em rede entre os grupos, pois os profissionais ganhariam maior volume de trocas interdisciplinares. Para a propagação dessas trocas e relacionamentos seria oportuno criar espaços para encontros temáticos para que ocorra diálogos entre os profissionais. É relevante que esses encontros, a partir de temas de interesse comum, sejam pautados, para incrementar trocas de saberes e informações entre os profissionais dos setores. Isso fortaleceria não só o relacionamento entre eles, como um meio para garantir a satisfação dos usuários atendidos.

Sugere-se como próximos passos realizar pesquisas longitudinais que possibilitem avaliar o desenvolvimento desta rede ao longo dos anos. Apesar deste estudo ter buscado desenvolver uma análise de redes da instituição de educação e saúde, ele tem limites, uma vez que não explorou toda as possibilidades das relações entre os agentes na sua gestão.

\section{Referências}

Acioli, S. (2007) Redes Sociais e Teoria Social: Revendo os Fundamentos do Conceito. Inf. Inf. Londrina, v. 12, n. esp.

Bufrem, L., \& Prates, Y. (2005). O saber científico registrado e as práticas de mensuração da informação. Ciência da Informação, 34(2), 9-25. Disponível em: <http://www.scielo.br/pdf/ci/v34n2/28551 >. Acesso em: 22 jan. 2018.

Carley, K. M. (2018) ORA-NetScenes Quick Start Guide, 2011 - 2013. Disponível em: $<$ http://www.casos.cs.cmu.edu/projects/ora/software/ORA\%20QuickStart\%20\%20v2.pdf $>$. Acesso em: 20 jan.

Cordeiro, M. P. (2009). Bibliometria e Análise de Redes Sociais: Possibilidades Metodológicas para a Psicologia Social da Ciência. Gerais: Revista Interinstitucional de Psicologia, 2(1), 23-33.

Fialho, J.; Moreno, R. (2015). O trabalho no setor bancário. Um estudo sobre a cooperação interorganizacional numa rede de agências bancárias. REDES- Revista hispana para el análisis de redes sociales. Vol. 26, n. 1, Junho.

Hayashi, C. M., Hayashi, M., Marcelo, J., \& Bello, S. (2012). Análise de redes de colaboração científica entre educação especial e fonoaudiologia. Revista Interamericana de Bibliotecología de Medellín, 35(3), 285-297.

INSTITUTE BRASILEIRO DE GEOGRAFIA E ESTATÍSTICA - IBGE. (2018). Projeção da população do Brasil e das Unidades da Federação. População do Brasil. Disponível em: $<$ https://www.ibge.gov.br/apps/populacao/projecao/ >. Acesso em: 20 jan.

Junqueira, L.A. P. (2000). Intersetorialidade, transetorialidade e redes sociais na saúde. Rio de Janeiro: RAP editora, p. 35-45. 
. A gestão da política de saúde e a conformação do Sistema Único de Saúde. (2006). In: Amorim, M.C.S.; Perillo, E.B.F. (org.) Para Entender a Saúde no Brasil. São Paulo: LCTE editora. .

PESQUISA NACIONAL DE SAÚDE - PNS (2013): Ciclos de Vida: Brasil e grandes regiões / IBGE, Coordenação de Trabalho e Rendimento. - Rio de Janeiro: IBGE, 2015. Disponível em < https://biblioteca.ibge.gov.br/visualizacao/livros/liv94522.pdf> Acesso em: 26 fev. 2018.

Maturana, H. R.; Varela, F. J. (2001). Árvore do Conhecimento: As Bases Biológicas da Compreensão Humana. São Paulo: Palas Athena.

Pinto, A. M. G. ( 2008). As relações de poder em redes do terceiro setor. 2008. Dissertação (Mestrado em Administração). Pontifícia Universidade Católica de São Paulo. São Paulo: PUCSP.

Rovere, M. (2018). Redes En Salud: Un Nuevo Paradigma para el abordaje de las organizaciones y la comunidad, Rosario: Ed. Secretaría de Salud Pública/AMR, Instituto Lazarte (reimpresión), 1999. Disponível em: <http://www.rosario.gov.ar/sitio/ verArchivo?id=8565\& tipo=objetoMultimedia $>$. Acesso em: 27 jan. 2018.

Sant'ana, L. (2011) Redes Sociais, Comunicação, Organizações. Análise de Redes Sociais como Metodologia para a Comunicação no contexto das organizações. Disponível em: <http://www.abrapcorp.org.br/anais2011/trabalhos/ trabalho_lidiane-santanna.pdf >. Acesso em: 27 jan. 2018.

Santanita, C. (2015). Dinâmicas intra-organizacionais num serviço em saúde, à luz da análise de redes sociais. REDES - Revista Hispana para el análisis de redes sociales. Vol. 26, n. 1.

Snijders, T. A. B. (2010). Actor-Based Models for Network Dynamics. Disponível em: <http://www.stats.ox.ac.uk/ snijders/PoliticalAnalysis_NetDyn.pdf>. Acesso em: 22 jan. 2018.

Teixeira, E. B.; Beber, M. C.; Grzybovski, D. (2008). Cooperação e Aprendizagem Organizacional em Organizações Sociais estruturadas em Redes de Cooperação: o caso da Rede Integração Social. XXXII Encontro da ANPAD. Anais. Rio de Janeiro-RJ, 06 a 10 de setembro de 2008.

Wasserman, S., \& Faust, K. (1994). Social Network Analysis: Methods and Applications. Social Networks (8). 